Departamento de Dermatología. Facultad de Medicina, Pontificia Universidad Católica de Chile, Santiago de Chile. aResidente de Dermatología.

Los autores declaran no haber recibido ningún tipo de apoyo financiero y la ausencia de conflictos de interés en el desarrollo de este artículo.

Recibido el 3 de noviembre de 2015, aceptado el 4 de enero de 2016.

Correspondencia a: Maximiliano Curi-Tuma Av. El Bosque Sur 131, depto. 53, Providencia, Santiago de Chile. maximilianocuri@gmail.com

\section{Actualización en técnica aséptica y uso de antibióticos profilácticos en procedimientos quirúrgicos ambulatorios que comprometan piel y mucosas}

\author{
MARCELA CONCHA-ROGAZY, CATALINA ANDRIGHETTI-FERRADA a \\ MAXIMILIANO CURI-TUMA ${ }^{\mathrm{a}}$
}

Aseptic techniques are those practices designed to reduce the risk of surgical site infection (SSI), defined as such, all those that occur within the first 30 days of the procedure. While the patient's risk of developing an SSI in dermatologic surgery is low since many of the procedures are considered sterile, there are different factors associated with an increased or decreased risk of developing SSI. The characteristics of the surgical wound (such as involving infected or inflamed tissue or when breaks in the aseptic technique occur), patient characteristics (such as age, comorbidities, medication use and smoking) and procedure factors (such as setting, surgical technique, type of procedure, duration and body region involved). In this article we discuss the management of potential sources of infections such as personnel (hand washing, dressing), preparation of the patient, maintenance of a clean surgical environment, sterilization and the use of antiseptic solutions. Similarly, the current indications for prophylactic antibiotics for these procedures are considered.

(Rev Med Chile 2016; 144: 1038-1043)

Key words: Asepsis; Dermatologic Surgical Procedures; Surgical Wound Infection.

L a técnica aséptica incluye todas aquellas prácticas diseñadas para reducir el riesgo de infección del sitio quirúrgico $(\mathrm{SSI})^{1}$. Se ha definido como infección de la herida operatoria ${ }^{2}$ (piel, tejido subcutáneo, músculo sobre la fascia en el sitio de incisión) a aquella que ocurre dentro de los primeros 30 días de realizado el procedimiento más uno de los siguientes:

- Drenaje purulento.

- Cultivo positivo (> 100.000 colonias).

- Al menos uno de los siguientes: dolor o sensibilidad, aumento de volumen, eritema o aumento de temperatura local.

- Diagnóstico de SSI hecho por cirujano o tratante.
El cuerpo humano está colonizado por microorganismos que se llaman colectivamente flora normal. En la piel, las características de esta colonización varía entre las distintas regiones corporales y está determinada por diferentes características, entre las que destaca condiciones de humedad y de contenido lipídico. La flora cutánea puede ser dividida en dos poblaciones distintas, la flora residente y la flora transitoria ${ }^{1}$.

Flora residente: Son microorganismos que pueden ser aislados en número similar en la mayoría de individuos. Corresponden a microorganismos comensales que protegen al huésped de infecciones al competir por sustratos y receptores 
tisulares. Se encuentran firmemente adheridos, por lo que son resistentes a la remoción mecánica y están lejos del alcance de soluciones antisépticas. Una de las metas de la técnica aséptica tiene relación con disminuir al mínimo posible la flora residente ${ }^{1}$.

Forman parte de esta flora:

- Staphylococci coagulasa (-) (S. epidermidis $>90 \%$ de residentes aerobios).

- Difteroides anaerobios (P. acnes).

- Gram (-): pequeña porción en áreas intertriginosas húmedas (Enterobacter, Klebsiella, E. coli, Proteus).

Flora transitoria: Es aquella población de microorganismos adquiridos a través del contacto con personas, objetos o el ambiente. Se encuentran pobremente adheridos a la piel, por lo que son susceptibles de remoción por lavado. Causan la mayoría de las SSI, siendo la fuente principal la flora endógena nasofaríngea o cutánea del paciente. Existen también fuentes exógenas como el personal de salud, instrumentos y el material quirúrgico ${ }^{1}$.

Forman parte de esta flora

- S. aureus: es el agente más frecuente de infección de sitio quirúrgico (SSI).

- Otros: estafilococos coagulasa negativo, Enterococo spp, E. coli, estreptococo grupo A y Pseudomona aeruginosa.

Las vías de transmisión más importantes en el ambiente quirúrgico son el contacto y el aerotransporte.

\section{Riesgo de infección de una herida quirúrgica}

Para poder establecer el riesgo que tiene una herida quirúrgica de infectarse es necesario considerar el tipo de herida operatoria, factores del paciente y factores de la cirugía ${ }^{3}$.

\section{A) Respecto a los tipos de heridas, pueden clasificarse según el grado de contaminación de la misma ${ }^{4}$}

Herida limpia: Incisiones electivas en tejidos no inflamados realizadas con técnica aséptica y que no involucra tracto gastrointestinal (GI), respiratorio o genitourinario $(\mathrm{GU})$. Tienen un riesgo bajo de infección menor a 5\%. En cirugía dermatológica, la gran parte de los procedimientos se consideran estériles y las heridas productos de los mismos, limpias.

Herida limpia contaminada: Son aquellas en las cuales existieron quiebres menores en la técnica aséptica o incisiones que involucran tracto GI, respiratorio o GU. Tienen un riesgo de infección cercano a $10 \%$.

Herida contaminada: Se consideran contaminadas aquellas heridas operatorias en cuyo procedimiento existieron quiebres mayores en técnica aséptica o se encuentran en un sitio inflamado pero sin purulencia franca. Tienen un riesgo de infección que va entre 20 y $30 \%$.

Herida sucia: Heridas que contienen fluido purulento como un absceso o involucra perforación visceral o contaminación fecal. Riesgo alto de infección, 30 a $40 \%$.

B) Características del paciente que influyen en el riesgo de desarrollar una infección de herida operatoria

Se deben considerar ${ }^{3}$ :

- Edad del paciente: existe un mayor riesgo de SII a mayor edad del paciente.

- Comorbilidades: se han relacionado con mayor riesgo fundamentalmente: diabetes mellitus descompensada, malnutrición, obesidad, insuficiencia renal crónica e inmunocompromiso.

- Respecto a medicamentos, el uso de corticoides y otros medicamentos inmunosupresores determinarían un mayor riesgo de SSI.

- Existe evidencia mixta en torno a tabaquismo, algunos estudios evidenciaron mayor riesgo de SSI en pacientes tabáquicos, lo que no ha sido constante en otros estudios. De igual forma, la alteración producida a nivel de curación de heridas secundaria al hábito tabáquico constituye argumento suficiente para recomendar a pacientes que no deben fumar.

- Se ha demostrado que pacientes portadores de $S$. aureus a nivel nasal desarrollan más SSI.

\section{C) Características de la cirugía}

La mayor parte de los estudios en cirugía se han realizado en un ambiente hospitalario. Faltan estudios para poder comparar riesgo de SSI en cirugías llevadas a cabo en un centro ambulatorio (mayoría de procedimientos dermatológicos) en relación al ambiente hospitalario. 
Tabla 1. Clasificación de heridas según nivel de contaminación y recomendación de profilaxis antibiótica en procedimientos quirúrgicos que comprometan piel y mucosas ${ }^{13}$

\begin{tabular}{|clll|}
\hline Clase & Condición de la piel & Localización/Técnica quirúrgica & Recomendación de profilaxis \\
\hline I & Limpia & Procedimiento estéril & No $(<5 \%)$ \\
II & Limpia/Contaminada & $\begin{array}{l}\text { Quiebres menores técnica estéril. Mucosa } \\
\text { oral, nasal, perineal o axila con procedimiento } \\
\text { estéril }\end{array}$ & $\begin{array}{l}\text { Solo sí inmunocomprometido } \\
(10 \%)\end{array}$ \\
III & Contaminada & $\begin{array}{l}\text { Herida traumática, inflamación no purulenta; } \\
\text { Quiebres mayores técnica estéril }\end{array}$ & $\begin{array}{l}\text { Antibióticos terapéuticos } \\
\text { (20-30\%) }\end{array}$ \\
IV & Infectada & $\begin{array}{l}\text { Contaminación, tejido desvitalizado, contami- } \\
\text { nación con cuerpo extraño }\end{array}$ & $\begin{array}{l}\text { Antibióticos terapéuticos } \\
\text { (30-40\%) }\end{array}$ \\
\hline
\end{tabular}

Una buena técnica quirúrgica es crítica en la prevención de SSI. Implica una adecuada hemostasia, un correcto manejo de los tejidos, evitando la isquemia, remover tejido desvitalizado y evitar la presencia de cuerpos extraños (suturas pueden comportarse como cuerpos extraños, monofilamentos tendrían menor riesgo $)^{1}$.

En general, procedimientos más largos conllevan un mayor riesgo de infección ${ }^{3}$. Regiones corporales como extremidad inferior, especialmente periungueal, región inguinal y perianal, constituyen sitios de mayor colonización por microorganismos, lo que también favorecerían una mayor tasa de $\mathrm{SSI}^{5}$.

Respecto al tipo de procedimiento, se ha visto que existe un mayor riesgo de infección en cirugías de neoplasias, colgajos, injertos y en general en cirugías más largas. Por ende, se debiesen preferir reparaciones simples siempre que éstas sean posibles ${ }^{3}$.

\section{Fuentes de contaminación}

En los procedimientos quirúrgicos hay 4 fuentes posibles de contaminación: el personal, el ambiente quirurgico, el paciente y los instrumentos, siendo la flora normal del paciente el reservorio más frecuente de microorganismos.

\section{A) Del personal de salud}

En relación al lavado de manos, es considerada una de las medidas más importantes para disminuir las infecciones asociadas a las atenciones de salud, lo que también se ha establecido en el ambiente quirúrgico. Existe la alternativa de realizar el lavado de manos con antisépticos y agua, pero últimamente se ha establecido la efectividad de soluciones de alcohol con ingredientes activos como clorhexidina ( $\sin$ necesidad de agua).

Es importante que los dispensadores de agua sean operados con el pie y que la calidad bacteriológica del agua esté asegurada. En relación al tiempo de lavado, éste se establece actualmente en torno a 2 a 5 min con el uso de agentes combinados.

Una revisión de la literatura ${ }^{6}$ consideró estudios clínicos aleatorizados comparando antisepsis de manos quirúrgica de duración, métodos y soluciones antisépticas variables, y estableció que:

- Lavado con clorhexidina es más efectivo en reducir la cantidad de bacterias en comparación con povidona yodada.

- No hay diferencias entre el uso de soluciones alcohólicas que contienen ingredientes activos y el lavado con soluciones antisépticas y agua en reducir SSI (evidencia mixta).

Respecto al vestuario del personal médico, no existe evidencia clara que el uso de ropa de pabellón, mascarilla, calzado quirúrgico, gorro o delantal estéril representen un beneficio en relación a obtener menores tasas de SSI. El uso de guantes estériles disminuiría SSI en casos complejos, lo que no está claramente establecido para reparaciones simples ${ }^{3}$. De igual forma, el uso de gorro, mascarilla, pechera y guantes contribuyen a un ambiente quirúrgico limpio por lo que se recomienda su uso.

\section{B) En relación a la preparación del paciente}

No existe evidencia concluyente del beneficio del uso de batas de algodón comparado con la ropa 
del paciente en términos de reducción de $\mathrm{SSI}^{1}$. La evidencia ha demostrado que afeitar el pelo del sitio quirúrgico se asocia a más infecciones, ya que podría inducir microabrasiones en piel que servirían de puerta de entrada a microorganismos y generaría ambiente inflamatorio pro-infeccioso. Se recomiendan otros métodos de remover el pelo (clips, geles depilatorios) cuando sea necesario hacerlo ${ }^{7}$.

No existe tampoco evidencia clara del beneficio del baño preoperatorio con clorhexidina por parte del paciente (día o noche anterior) versus otros productos de baño para reducir SSI ${ }^{8}$.

\section{C) Respecto a la mantención de un ambiente quirúrgico limpio}

Es importante mantener las puertas del pabellón bien cerradas y restringir el flujo de personas en el pabellón. Estas medidas impiden la reaerosolización de partículas y la eventual contaminación del campo quirúrgico ${ }^{1}$. Además, existen protocolos de limpieza y desinfección de pabellones, que incluye limpieza terminal y desinfección con amonio cuaternario al final del día.

\section{D) La esterilización del material}

Es necesaria para destruir todas las formas de microorganismos viables, incluyendo las esporas ${ }^{1}$. En nuestro medio, lo más utilizado es el autoclave, un sistema de calor húmedo a presión, que permite esterilizar la mayor parte del material, exceptuando plásticos. Es un procedimiento económico, eficiente y de fácil monitorización. Otras formas de esterilización son el vapor químico caliente, el calor seco, la esterilización con gas (como óxido de etileno) y la inmersión química.

\section{Antisépticos y piel}

Se consideran antisépticos aquellos agentes químicos que son capaces de eliminar microorganismos de la piel y mucosas?.

El antiséptico ideal es aquel de amplio espectro, de inicio rápido de acción, con efecto residual y baja toxicidad. Debe ser estable, no inactivarse frente a la presencia de materia orgánica ni por factores del ambiente. Deseablemente, su costo debe ser razonable.

Uno de los antisépticos más usados es la povidona yodada 9 . Posee un amplio espectro de acción frente a bacterias, virus, hongos, protozoos, quistes, amebas y esporas. Ejerce su acción a través de oxidación e inactivación de componentes celulares, destruye citoplasma y membrana celular. Se deben esperar 2-3 min para que comience a actuar, y horas para ejercer su acción sobre esporas secas. Tiene efecto residual por acción del yodo (aproximadamente $3 \mathrm{~h}$ ). Se inactiva con sangre y materia orgánica. Presenta toxicidad sistémica en recién nacidos (riesgo de hipotiroidismo), no debe ser usada en embarazadas. De elección en región periocular y pabellones auriculares.

Otro antiséptico de uso extendido es la clorhexidina, generalmente en su forma de gluconato ${ }^{9}$. También posee un amplio espectro con acción sobre bacterias Gram (+) y Gram (-), virus como $\mathrm{VIH}$, herpes simple, citomegalovirus e influenza. No tiene acción sobre el bacilo tuberculoso y posee una acción débil en hongos. Actúa sobre membrana celular y genera precipitación del citoplasma. Presenta un inicio rápido de acción y un efecto residual cercano a las $6 \mathrm{~h}$ (se adhiere al estrato córneo). No se neutraliza en presencia de materia orgánica. Bien tolerado en general, debe evitarse su uso en región periocular (irritación, conjuntivitis, riesgo de úlcera corneal) y en pabellones auriculares por presentar ototoxicidad. Las formulaciones más comunes son a $2 \%$ y $4 \%$.

El alcohol (70\% o concentraciones superiores) es un antiséptico con un amplio espectro (formas vegetativas de Gram [+] y [-], bacilo tuberculoso, hongos y virus [VHB, VIH]), actúa desnaturalizando las proteínas. No tiene acción detergente, por lo que debe aplicarse sobre la piel previamente limpia. Presenta un rápido inicio de acción, pero carece de actividad residual, por lo que se suele combinar con otro agente (como gluconato de clorhexidina $)^{9}$. Luego de su aplicación hay que esperar a que se seque. Muy inflamable si esta húmedo todavía, por lo que puede constituir un riesgo en pabellón. Existen reportes de casos de quemaduras de distinta gravedad por el uso de antisépticos que contienen alcohol en procedimientos que implican el uso de electrobisturí ${ }^{10}$.

\section{$\underline{\text { IV. Uso de antibióticos en cirugía dermatológica }}$}

El uso de antibióticos en dermatología puede darse en una situación de profilaxis (de infección de herida operatoria o de endocarditis infecciosa/infección prótesis articular en pacientes de 
alto riesgo) o para el tratamiento de una herida infectada ${ }^{11}$.

La mayor parte de las SSI resultan de flora residente presente en epidermis o mucosas al momento de la incisión. El que esa contaminación resulte en infección depende de una compleja interacción entre el huésped y el microorganismo ${ }^{11}$.

En caso de que exista indicación de antibióticos profilácticos, es importante considerar el o los agentes posiblemente involucrados en la infección que se quiere prevenir. Esto tiene relación con sitio de cirugía (por ejemplo, en procedimientos en cavidad oral existe riesgo de infección por estreptococo beta-hemolítico de grupo A, S. aureus o $S$. viridans) o microorganismos que pudiesen causar el cuadro que quisiera evitarse (por ejemplo $S$. viridans para endocarditis infecciosa $)^{3}$.

El uso de antibióticos profilácticos en cirugía dermatológica no está indicado de rutina principalmente porque la incidencia de infecciones posteriores a los procedimientos es baja. A esto se agregan posibles eventos adversos producto de la administración de antibióticos (en cualquiera de sus vías de administración), la emergencia de mircoorganismos resistentes y los costos asociados al uso de estos ${ }^{12}$.

El riesgo de bacteremia en procedimientos dermatológicos en piel intacta y limpia es menor a $2 \%$, incluso en procedimientos complejos y largos, por lo tanto, el riesgo de infección de una prótesis articular o el desarrollo de una endocarditis infecciosa es bajo. Este riesgo es mayor si el sitio quirúrgico es en mucosa ${ }^{5,12}$.

En general, heridas limpias no necesitan profilaxis antibiótica. Cirugías que involucren mucosa oral o nasal, el area axilar o anogenital debería recibir profilaxis si el paciente es inmunocomprometido. Si hay un quiebre mayor en la técnica estéril o una herida francamente inflamada o infectada, se requieren antibióticos terapéuticos, considerando la región comprometida ${ }^{3,4}$.

Se han determinado factores de riesgo del paciente, ya mencionados, y otros asociados al procedimiento/área anatómico para desarrollar $\mathrm{SSI}^{3,5}$ :

Factores de riesgo asociados al procedimiento y área anatómica:

- Cirugías o procedimientos bajo la rodilla e inguinales.

- Colgajos, incisiones en cuña y colgajos de oreja y labio, cirugía en nariz.

- Enfermedad inflamatoria extensa.
Respecto a la evidencia existente en relación al uso de antibióticos profilácticos en cirugía dermatológica, no existe evidencia de una disminución de SSI con el uso de antibióticos tópicos (sin diferencias con el uso de vaselina tópica). Además, su uso indiscriminado contribuiría a la emergencia de microorganismos resistentes ${ }^{3}$.

Si bien existe un mayor riesgo de SII en pacientes portadores nasales de S. aureus, no hay estudios aleatorizados que prueben que el tratamiento de erradicación con mupirocina intranasal disminuya la tasa de infeccion de herida quirúrgica en portadores ${ }^{3}$.

Respecto al uso de antibióticos intraincisionales, existen estudios que han mostrado menos tasas de infección, y dada su menor absorción sistémica un mejor perfil de efectos adversos, pero faltan estudios para confirmar estos hallazgos ${ }^{3}$.

En relación a la indicación de antibioticoterapia oral profiláctica, se acepta como razonable su uso frente a procedimientos quirúrgicos de riesgo y en el caso de herida limpia contaminada en pacientes inmunosuprimidos. Estos antibióticos deben ser administrados previo al procedimiento (30 a 60 min antes) y se considera de elección una cefalosporina de primera generación (cefalotina en estudios internacionales, cefadroxilo en nuestro medio $)^{3,5}$. Se debe considerar la flora del sitio quirúrgico (por ejemplo, añadir ciprofloxacino o clindamicina para cubrir Gram negativos en región perineal o áreas intertriginosas). En pacientes con alto riesgo de endocarditis infecciosa o de infección de prótesis articular está indicada la profilaxis antibiótica en procedimientos que involucren mucosa oral (de elección amoxicilina) o un sitio francamente infectado ${ }^{3,5}$.

\section{Referencias}

1. Ammirati C. Aseptic Technique. In: Surgery of the Skin: Procedural Dermatology. Chapter 2: 25-35.

2. Horan TC, Gaynes RP, Martone WJ, Jarvis WR, Emori TG. CDC definitions of nosocomial surgical site infections, 1992; A modification of CDC definitions of surgical wound infections. Infection Control Hosp Epidemiol 1992; 13: 606-8.

3. Rosengren H, Heal C, Smith S. An Update on Antibiotic Prophylaxis in Dermatologic Surgery. Curr Derm Rep 2012; Volume 1 (2): 55-66.

4. Yuste M, Romo A, de Unamuno P. Antibiotic pro- 
phylaxis in dermatologic surgery. Actas Dermosifiliogr 2008; 99 (9): 683-9.

5. Wright T, Baddour L, Berbari E, Roenigk R, Phillips P, Jacobs $\mathrm{M}$, et al. Antibiotic prophylaxis in dermatologic surgery: advisory statement 2008. J Am Acad Dermatol 2008; 59 (3): 464-73.

6. Tanner J, Swarbrook S, Stuart J. Surgical hand antisepsis to reduce surgical site infection. Cochrane Database of Systematic Reviews 2008, Issue 1.

7. Tanner J, Woodings D, Moncaster K. Preoperative hair removal to reduce surgical site infection. Cochrane Database of Systematic Reviews 2006; 3: 1-35.

8. Webster J, Osborne S. Preoperative bathing or showe- ring with skin antiseptics to prevent surgical site infection. Cochrane Database Syst Rev 2012.

9. http://www6.uc.cl/manejoheridas/html/antiseptico. html

10. Bonnet A, Devienne M, De Broucker V, Duquennoy-Martinot V, Guerreschi P. Operating room fire: Should we mistrust alcoholic antiseptics? Ann Chir Plast Esthet 2015; 60 (4): 255-61.

11. Haas A. Antibiotics. In: Surgery of the Skin: Procedural Dermatology. Chapter 9: 137-46.

12. Shurman DL, Benedetto AV. Antimicrobials in dermatologic surgery: facts and controversies Clin Dermatol 2010; 28 (5): 505-10. 